\title{
Intraspinal pressure and spinal cord perfusion pressure after spinal cord injury: an observational study
}

\author{
Georgios V. Varsos, MSc, ${ }^{1}$ Melissa C. Werndle, MRCS, ${ }^{2}$ Zofia H. Czosnyka, PhD, ${ }^{1}$ \\ Peter Smielewski, PhD, ${ }^{1}$ Angelos G. Kolias, MRCS, ${ }^{1}$ Isaac Phang, MRCS, ${ }^{2}$ \\ Samira Saadoun, PhD, ${ }^{2}$ B. Anthony Bell, MD, FRCS, ${ }^{2}$ Argyro Zoumprouli, MD, ${ }^{3}$ \\ Marios C. Papadopoulos, MD, FRCS(SN), ${ }^{2}$ and Marek Czosnyka, PhD ${ }^{1,4}$
}

'Division of Neurosurgery, Department of Clinical Neurosciences, Addenbrooke's Hospital, University of Cambridge, Cambridge; ${ }^{2}$ Academic Neurosurgery Unit, St. George's University of London; ' ${ }^{2}$ epartment of Anaesthesia, St. George's Hospital, London, United Kingdom; and ${ }^{4}$ Institute of Electronic Systems, Warsaw University of Technology, Warsaw, Poland

\begin{abstract}
OBJECT In contrast to intracranial pressure (ICP) in traumatic brain injury (TBI), intraspinal pressure (ISP) after traumatic spinal cord injury (TSCI) has not received the same attention in terms of waveform analysis. Based on a recently introduced technique for continuous monitoring of ISP, here the morphological characteristics of ISP are observationally described. It was hypothesized that the waveform analysis method used to assess ICP could be similarly applied to ISP. METHODS Data included continuous recordings of ISP and arterial blood pressure (ABP) in 18 patients with severe $\mathrm{TSCl}$.

RESULTS The morphology of the ISP pulse waveform resembled the ICP waveform shape and was composed of 3 peaks representing percussion, tidal, and dicrotic waves. Spectral analysis demonstrated the presence of slow, respiratory, and pulse waves at different frequencies. The pulse amplitude of ISP was proportional to the mean ISP, suggesting a similar exponential pressure-volume relationship as in the intracerebral space. The interaction between the slow waves of ISP and ABP is capable of characterizing the spinal autoregulatory capacity.

CONCLUSIONS This preliminary observational study confirms morphological and spectral similarities between ISP in TSCI and ICP. Therefore, the known methods used for ICP waveform analysis could be transferred to ISP analysis and, upon verification, potentially used for monitoring $\mathrm{TSCl}$ patients.
\end{abstract}

http://thejns.org/doi/abs/10.3171/2015.3.SPINE14870

KEY WORDS intraspinal pressure; spinal cord injury; biophysics; observation

$\mathrm{U}$ NDER normal circumstances, spinal CSF offers protection to the spinal cord by acting like a shockabsorbing mechanism. However, after an acute traumatic spinal cord injury (TSCI) when the injured section of the spinal cord is swollen, there is no or a minimal amount of CSF between the neural tissue and the surrounding dura mater. This situation shares some characteristics with a swollen brain: the lack of CSF may decrease the local pressure-volume compensatory reserve and, in cases of further swelling or volume expansion, produce a rapid local rise in intraspinal pressure (ISP) within the injured compartment, as the low-distensible dura is unable to provide any CSF buffering. This, in turn, may produce intraspinal hypertension, thereby producing irreversible local spinal cord ischemia that leads to neuronal death. In traumatic brain injury (TBI), increased intracranial pressure (ICP) may impede cerebral blood flow as it reduces cerebral perfusion pressure (CPP), which is calculated as the difference between arterial blood pressure (ABP) and ICP. ${ }^{8,14,16,21}$ Similarly, ISP affects the spinal cord perfusion pressure (SCPP), which is the difference between $\mathrm{ABP}$ and ISP. ${ }^{25}$ Therefore, knowledge of ISP behavior in such

ABBREVIATIONS ABP = arterial blood pressure; CPP = cerebral perfusion pressure; ICP = intracranial pressure; ISCoPE = Injured Spinal Cord Pressure Evaluation; ISP $=$ intraspinal pressure; $\mathrm{PRx}=$ pressure reactivity index; $\mathrm{SCPP}=$ spinal cord perfusion pressure; $\mathrm{sPRx}=\mathrm{spinal} \mathrm{PRx} ; \mathrm{TBI}=$ traumatic brain injury; $\mathrm{TSCl}=$ traumatic spinal cord injury.

SUBMITTED August 22, 2014. ACCEPTED March 25, 2015.

INCLUDE WHEN CITING Published online August 14, 2015; DOI: 10.3171/2015.3.SPINE14870. 
situations can provide valuable information concerning the condition of the spinal cord in cases of TSCI.

While waveform analysis of ICP has been conducted for years and has been proven to be helpful in the management of TBI patients, ${ }^{15}$ very little is known about ISP in the acute phase following TSCI where its management focuses on spine realignment and fixation without taking into account ISP. ${ }^{25}$ The underlying reason behind this is the absence of a safe and reliable long-term monitoring technique for measuring ISP, because techniques such as Queckenstedt's maneuver, which was being used to examine spinal fluid dynamics in TSCI, were replaced by imaging modalities (e.g., MRI or CT scanning) which in turn are not suitable for long-term monitoring. ${ }^{22}$ This issue was addressed recently with the introduction of a novel technique for continuously monitoring ISP at the injury site, which has allowed discussions on using ISP for managing TSCI in a similar way that ICP is used to manage TBI. ${ }^{25}$ Based on the abovementioned similarities between the injured brain and spinal cord, it is vital to recapitulate how the information included in the time-dependent components of ICP is crucial for understanding intracranial pathophysiology. ${ }^{12}$ This may form the basis for similar analyses on monitoring ISP.

ICP is derived from 3 main components: the circulation of the CSF and cerebral blood, and volumetric changes in brain tissue. ${ }^{9}$ According to Davson's equation, ${ }^{13}$ the component associated with CSF circulation is also related to cerebral venous outflow. Expressing the cerebral blood component quantitatively has been far more difficult. It has been defined as the "vascular component" of the ICP waveform ${ }^{19}$ and is known to be related to cerebral blood volume and arterial blood pulsatile flow. ${ }^{11,19}$ Any factor that disturbs the CSF or cerebral blood circulation, like brain swelling or space-occupying mass lesions, may result in increases in ICP.

The waveform of ICP consists of slow fluctuations and regular respiratory and pulse waves that are seen at different frequencies (low to high frequency, respectively). ${ }^{3,9}$ Its pulse waveform contains 3 peaks-percussion, tidal, and dicrotic-with the relationship between the tidal and percussion peaks describing brain compliance. ${ }^{5}$

Secondary indices of ICP can be descriptive of the cerebrospinal compensatory reserve (RAP index), or-based on the interactions between the slow waves of ICP and $\mathrm{ABP}$ - can characterize the status of the cerebrovascular pressure reactivity (PRx index). ${ }^{3,6-8,10,24}$ In TBI cases, both ICP and its derived indices are well known to have a strong association with outcome and are part of the clinical assessment for deciding the therapeutic management of a patient. ${ }^{3,8,9,15,16,24}$

Based on the inherent similarities with ICP, this observational study aims to qualitatively describe the biophysics of ISP and explore its morphology regarding different components in the time and frequency domains.

\section{Methods}

This retrospective study included prospectively collected data from 18 patients (age range 18-70 years) with severe TSCI (American Spinal Injury Association Grades
A-C) who were referred to the Neurosurgery Units of St. George's and King's College hospitals in London and recruited to participate in the ISCoPE (Injured Spinal Cord Pressure Evaluation) study. ${ }^{25}$ Approval was obtained from the local research ethics committee at St. George's Hospital in London. The details of the ISCoPE study have been described before ${ }^{25}$ and are briefly recapitulated here.

For ISP measurement, a Codman probe was placed subdurally following laminectomy or small laminotomy. Monitoring of ISP started within 72 hours of TSCI and continued for up to a week. In regards to the placement of the probe, after reducing and fixing the spinal fracture and inserting metalwork to stabilize the spine, a 14-gauge introducer was used to tunnel the Codman probe through the skin into the wound. A 21-gauge needle, which was bent at $90^{\circ}$, was used to perforate the dura at 1 level below the injury. To monitor ISP, the Codman probe was calibrated and advanced through the dural hole until the probe tip was at the site of maximal spinal cord swelling according to the MRI scan, and satisfactory probe position was confirmed with CT before data collection. ${ }^{25}$ In 4 patients, additional probes were inserted for simultaneous monitoring of both subdural and extradural pressure.

Regarding the effect of therapeutic interventions on ISP, changes in arterial $\mathrm{PCO}_{2}$, sevoflurane dose, and mannitol administration were not found to have any significant effects on ISP or SCPP. Increasing the inotrope dose significantly increased SCPP. ${ }^{25}$ Bony realignment and laminectomy did not effectively lower ISP, with laminectomy being potentially detrimental by exposing the swollen spinal cord to compression forces applied to the skin. ${ }^{25}$

Imaging assessment of ISP was not studied in this cohort because, as in TSCI, spinal cord imaging may be uninterpretable because of spinal instrumentation, thus making it difficult to use imaging examinations to guide TSCI management. ${ }^{25}$

ABP was measured using a radial artery catheter that was calibrated at the same horizontal level as the injured segment of the spinal cord. Probes were connected to a Codman ICP box linked via a ML 221 amplifier to PowerLab running LabChart (v. 7.3.3, AD Instruments). Further details regarding the clinical methods and surgical technique were recently published. ${ }^{25}$ ISP and ABP data were simultaneously captured at $100 \mathrm{~Hz}$ and then imported to ICM+ software (Cambridge Enterprise; http://www.neuro surg.cam.ac.uk/icmplus/) for further analysis.

Visual inspection, time averaging, and spectral analysis were performed to assess similarities in the ISP waveforms to known ICP recordings determined by our previous studies. ${ }^{3,6-8,9,12}$

\section{Analysis of the ISP Waveform}

The ISP waveform can be analyzed by focusing on the signal's time evolution in the respective monitoring period. Monitoring can last from seconds to hours or days; however, like ICP, ${ }^{18}$ ISP can be very unstable, and, therefore, longer monitoring periods are essential in order to gain a clear idea of the ISP trends. In that case, averaging the ISP signal over a short period of time (e.g., a 10-second time window) can provide a smooth trend for the mean ISP that has been filtered for noisy signals. The same logic 
applies to ABP, and a relevant time window is needed to determine the average value of the signal. SCPP is calculated as ABP minus ISP. Important fluctuations, like slow waves and respiratory and pulse waves, can be visualized using spectral analysis of the frequency domain and shortterm fast Fourier transform. The amplitude of ISP (I1) is derived from the fundamental component (first harmonic) of the pulse wave in the ISP signal.

\section{sPRx and sRAP Indices for ISP}

In TBI, analysis of the ICP waveform using the PRx and RAP indices can aid in the assessment of both cerebrovascular reactivity status and cerebrospinal compensatory reserve, respectively. PRx reflects the ability of the vascular smooth muscle to respond to changes in transmural pressure and is used as a surrogate index of autoregulation., ${ }^{9,24}$ PRx is calculated as a moving correlation coefficient between the slow waves of ICP and ABP on a 5-minute sliding window. ${ }^{89}$ A positive PRx $(>0.3)$ is known to be associated with an unfavorable outcome after TBI. . $^{3,8,9,24}$

The relationship between PRx and CPP demonstrates a U-shaped curve in TBI and SAH, which is known to describe the failure of autoregulation that occurs at both very low and very high CPP., ${ }^{9,24}$ This relationship has given birth to the concept of "optimal CPP," or the CPP value at which cerebral autoregulation is the strongest and indicated by the lowest PRx value on the patient's U-shaped curve.1,24 A CPP that is much higher or much lower than the optimal CPP has been suggested to be associated with an unfavorable outcome following TBI. ${ }^{24}$

The RAP (where $\mathrm{R}$ represents the correlation coefficient; $\mathrm{A}$, the amplitude; and $\mathrm{P}$, the mean pressure) index represents the dynamic pressure-volume relationship inside the cerebrospinal space, indicating whether the compensatory reserve is intact or exhausted. RAP can be calculated in a similar way as PRx, i.e., as the Pearson's moving correlation coefficient between changes in amplitude and mean ICP. ${ }^{10}$ An RAP around 0 at low $\mathrm{ICP}(<15 \mathrm{~mm} \mathrm{Hg})$ indicates good cerebrospinal compensatory reserve, whereas RAP close to +1 indicates an exhausted compensatory reserve. Lower RAP values (i.e., negative values) at increased ICP (> $20 \mathrm{~mm} \mathrm{Hg}$ ) are associated with unfavorable outcome in TBI patients, ${ }^{6,10}$ indicating terminal cerebrovascular derangement., 3

Due to the similarities between ICP and ISP, the PRx and RAP indices might also be used to assess SCI in the same way that they are used in TBI, potentially providing some useful indications concerning autoregulation and compensatory reserve. ${ }^{25}$

Using the same methodology, the equivalent indices are now called spinal PRx (sPRx) and spinal RAP (sRAP). To calculate sPRx, instead of correlating ABP and ICP, we substitute the latter with ISP. Thirty consecutive 10 -second averages of ISP, ABP, and the pulse amplitude of ISP are taken to calculate the moving correlation coefficients within a 5-minute window. We hypothesized that sPRx would indicate intact or disturbed cerebrospinal reactivity, showing negative or positive values, respectively, and that the status of the local spinal compensatory reserve at the injury site of a TSCI could be described by the sRAP index.

\section{Results \\ Morphology of the Peaks of the ISP Pulse Waveform}

Analogous to ICP, 3 peaks appear in the ISP pulse waveform-P1, P2, and P3 - as demonstrated in Fig. 1. P1 is associated with the systolic peak of $\mathrm{ABP}$, representing the percussion wave. P2 corresponds to intraspinal compliance, representing the tidal wave. P3 is linked to aortic valve closure and the dicrotic wave subsequent to the dicrotic notch of arterial pulsation.

Elevation of the mean ISP alters the shape of the pulse waveform: as ISP starts to increase, P2 also rises with the pulse waveform, acquiring a round shape due to the superimposition of $\mathrm{P} 1$ and $\mathrm{P} 2$; the difference between them becomes hardly recognizable (Fig. 1A). As ISP continues to increase, $\mathrm{P} 2$ rises further to become clearly distinguishable on the pulse waveform at the level above P1 and P3 (Fig. 1B). At very high ISP, there is a disproportionate elevation of P2 and P3, with the pulse waveform acquiring a triangular or pyramidal shape where $\mathrm{P} 2$ is dominant and P1 is hardly visible (Fig. 1C).

\section{Time and Frequency Domains}

The ISP waveform contains 3 wave components that overlap in the time domain. Corresponding spectral analysis of the frequency domain allows the separation of these components, which can be identified as slow, respiratory, and pulse waves (Fig. 2). Slow waves are present at low frequencies $(0.05-0.0055 \mathrm{~Hz}$, or in periods of 20 seconds to 3 minutes), whereas respiratory waves are related to the frequency of the respiratory cycle at $0.13-0.3 \mathrm{~Hz}$ or $8-20$ cycles/minute. The pulse waves consist of different harmonics, with the first harmonic denoted as the fundamental component corresponding to the frequency equal to a heart rate $(\sim 1 \mathrm{~Hz})$, while higher peaks in the frequency domain are considered higher harmonics.

\section{Relationship Between Mean ISP and Its Amplitude}

A typical trend for the relationship between mean ISP and its fundamental harmonic amplitude (I1) is presented in Fig. 3 as a long-term recording from a single patient. The 2 parameters-ISP and I1-demonstrate a positive and direct relationship, and increasing ISP leads to increases in I1. These proportional changes are similar to the ones described for the pulse amplitude of ICP caused by changes in mean ICP. $1,20,23$

The relationship between ISP and I1 is described by the time domain of the sRAP index, which can express the status of the spinal compensatory reserve: the example in Fig. 4 demonstrates the continuous 5-day monitoring of ISP and I1, with sRAP fluctuating from periods of low $(\sim 0)$ to high $(\sim 1)$ values, thereby signifying periods where pressure-volume compensation is normal or impaired, respectively.

\section{Slow ISP Waves and Interaction With ABP}

The interaction between the slow fluctuations of ABP and ISP is expressed by the sPRx index, the values of which can be indicative of the spinal vascular reactivity capacity. This capacity can be monitored, with the exam- 

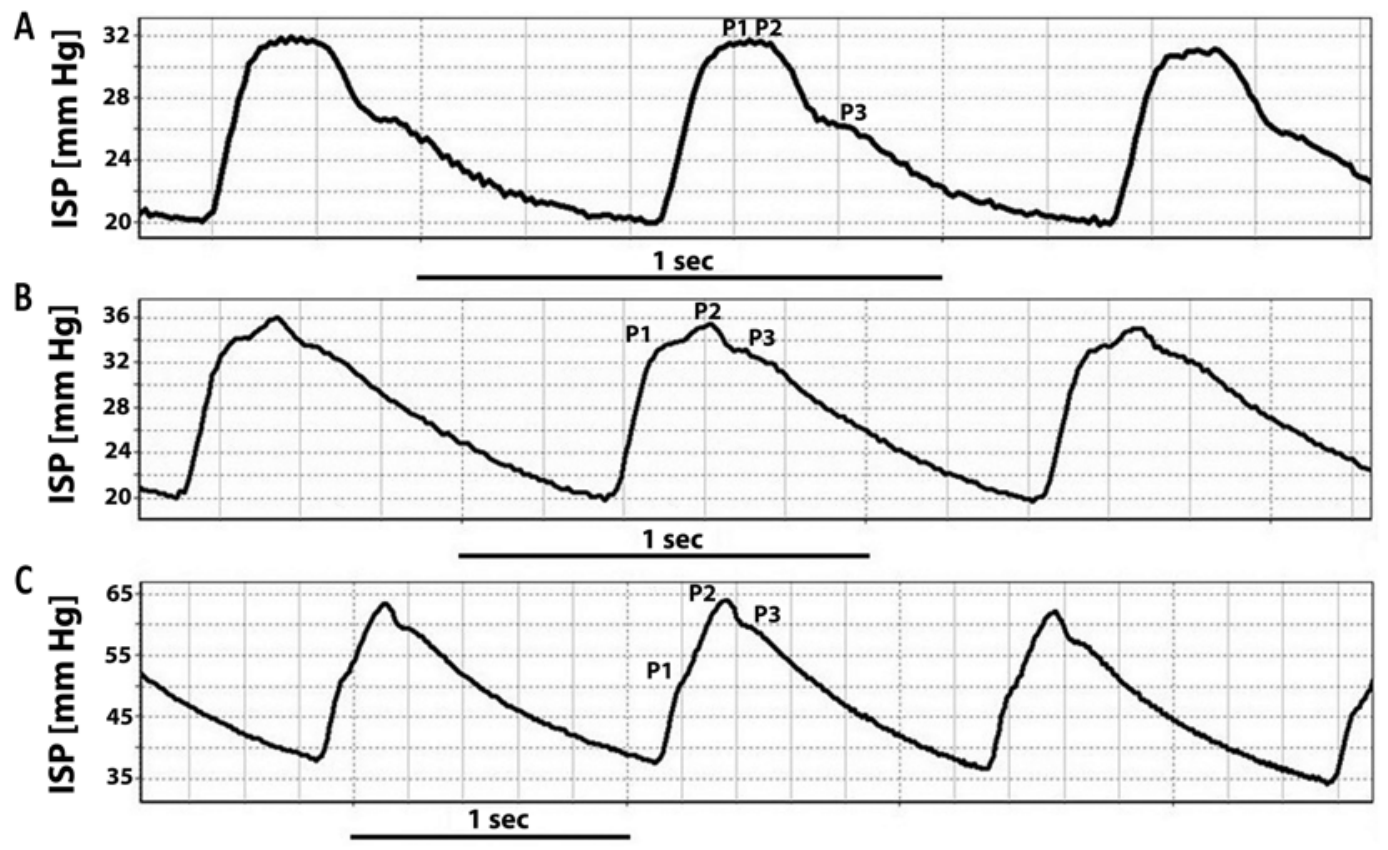

\section{Time (sec)}

FIG. 1. ISP pulse wave consists of 3 main components represented by 3 peaks: the percussion wave (P1); the tidal wave (P2); and the dicrotic wave (P3). The morphological shape of the ISP pulse wave is altered with different levels of ISP. A: Increased ISP results in an increased P2, which becomes superimposed with P1 and results in a round ISP waveform. B: ISP at a higher level has a clearly distinguishable P2 that is higher than the P1 and P3 levels. C: At very high ISP, the pulse wave acquires a triangular shape, and $\mathrm{P} 2$ becomes dominant and $\mathrm{P} 1$ becomes less visible.

ple included in Fig. 5 depicting long-term fluctuations in sPRx. In the beginning, sPRx is unstable and varies from highly positive to highly negative, but after the middle of the recording period it becomes stable and positive $(>0.4)$, thereby signifying impaired reactivity.

The examples in Fig. 6 demonstrate 2 contrasting cases of spinal reactivity capacity. In Fig. 6 left, sPRx is low and negative, indicating an inverse relationship between changes in ABP and ISP, and thus the presence of pres- sure reactivity. In contrast, Fig. 6 right depicts a highly positive sPRx, denoting a positive and passive relationship between ABP and ISP, therefore characterizing a depleted reactivity reserve.

\section{Relationship Between sPRx and SCPP}

Based on the similarities with PRx in terms of pressure reactivity, sPRx may as well characterize the equivalent spinal autoregulatory capacity. For this reason, we sought
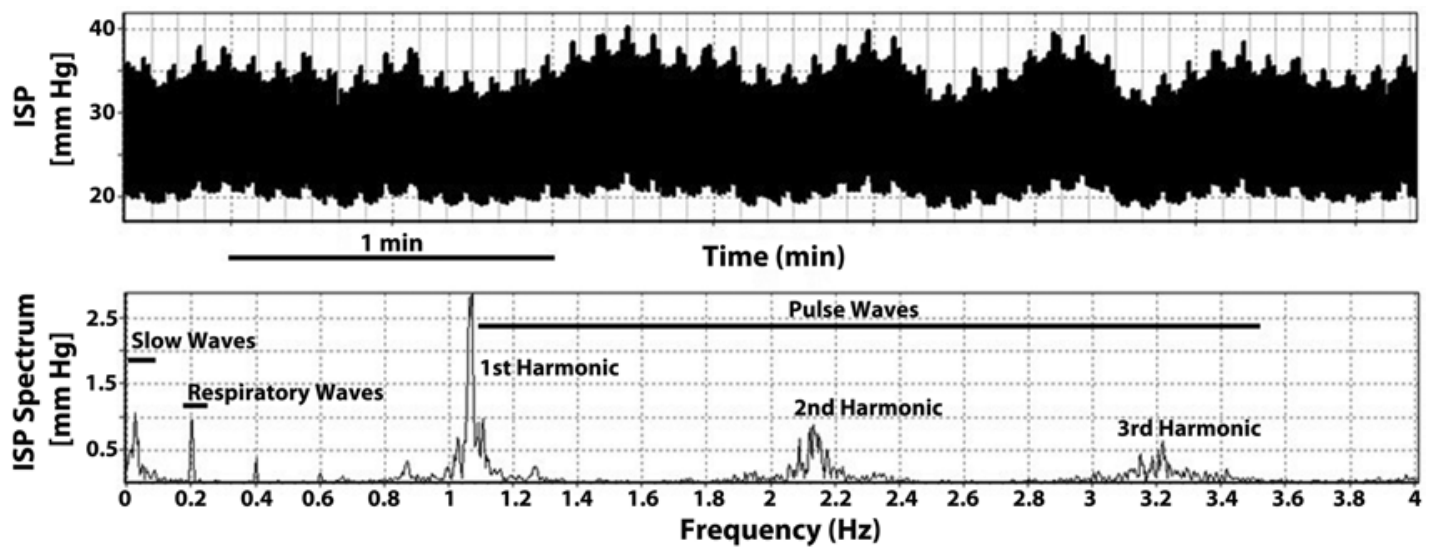

FIG. 2. Signal analysis of ISP in the time and frequency domains of a patient's recording. Spectral analysis of ISP demonstrates slow vasogenic waves at a low frequency, followed by respiratory waves and pulse waves at a higher frequency. The pulse waves consist of multiple harmonics, with the fundamental harmonic (1st harmonic) appearing at a frequency equivalent to a heart rate, followed then by second and third harmonics. A similar effect can be seen in higher harmonics of the respiratory wave. 

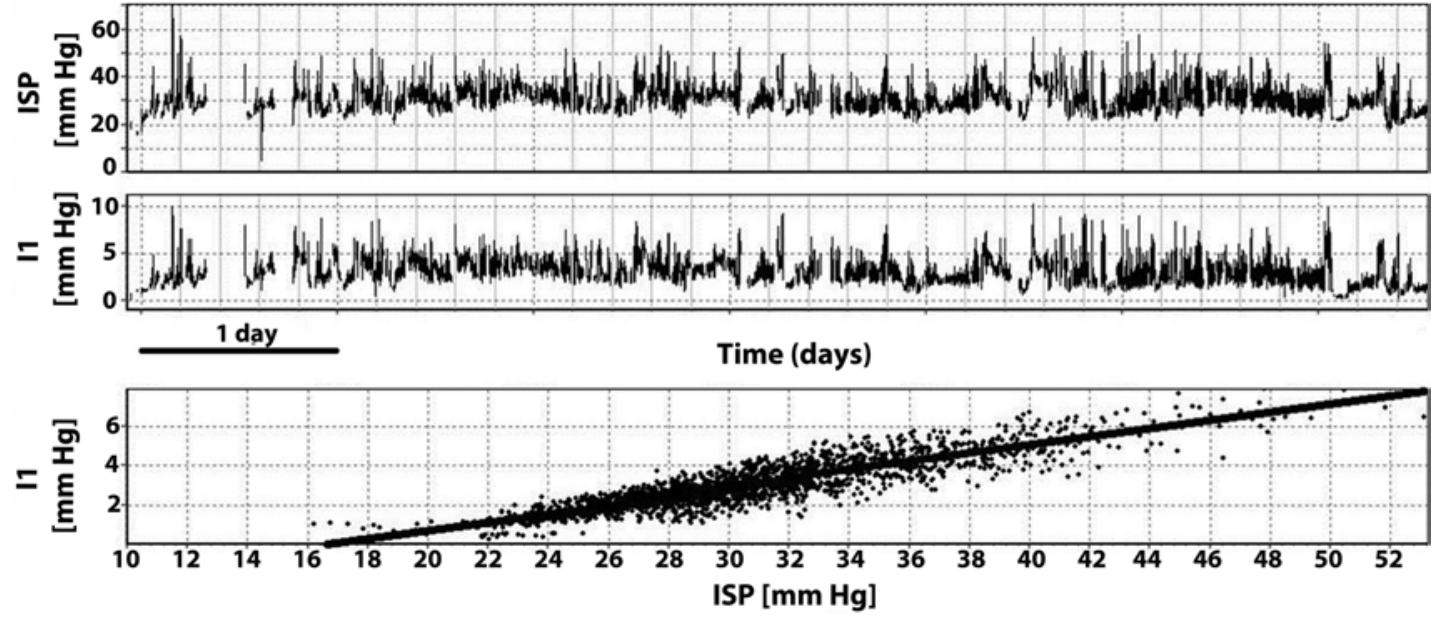

FIG. 3. Time series of ISP and its fundamental harmonic amplitude (I1) in a long-term recording of a single patient. The scatter plot demonstrates a positive relationship between I1 and ISP: rising ISP results in an increase in I1.

to explore its relationship with SCPP. A typical example is shown in Fig. 7, where the plot between the 2 parameters shows a U-shaped curve. This relationship illustrates that spinal autoregulation becomes worse at both low and high SCPP, as indicated by high sPRx at both occasions. The middle part of the curve signifies the optimum SCPP range for the best spinal autoregulation, which is based on low sPRx values. The lowest sPRx value indicates optimal SCPP.

\section{Relationship Between Subdural and Extradural Pressure}

Simultaneous monitoring of both subdural and extradural pressure allowed observational comparison of pressures at both sides of the dura mater (Fig. 8). Extradural pressure demonstrates similar behavior as ISP, however with some notable differences: 1) overall, extradural pressure is lower than ISP, with the difference being in the range of $2-3 \mathrm{~mm} \mathrm{Hg}$ as shown by the particular example in Fig. 8; 2) extradural pressure pulsatility seems to be lower than ISP with a smaller peak-to-peak amplitude; 3 ) extradural pressure demonstrates similar fluctuations to ISP, however the magnitude of the slow waves is visibly smaller. Overall, these remarks denote the buffering role of the dura, with the characteristics of extradural pressure being similar, but attenuated, in comparison with the characteristics of ISP.

\section{Discussion}

The first paper to describe ISP recordings was recently published, ${ }^{25}$ with the use of a microsensor inserted in the operating room allowing continuous and reliable ISP recordings that lasted up to a few days. This new and promising technique has allowed us to observe in close detail the morphology of the ISP pulse waveform and its different wave components.

The ISP waveform resembles the respective ICP wave-

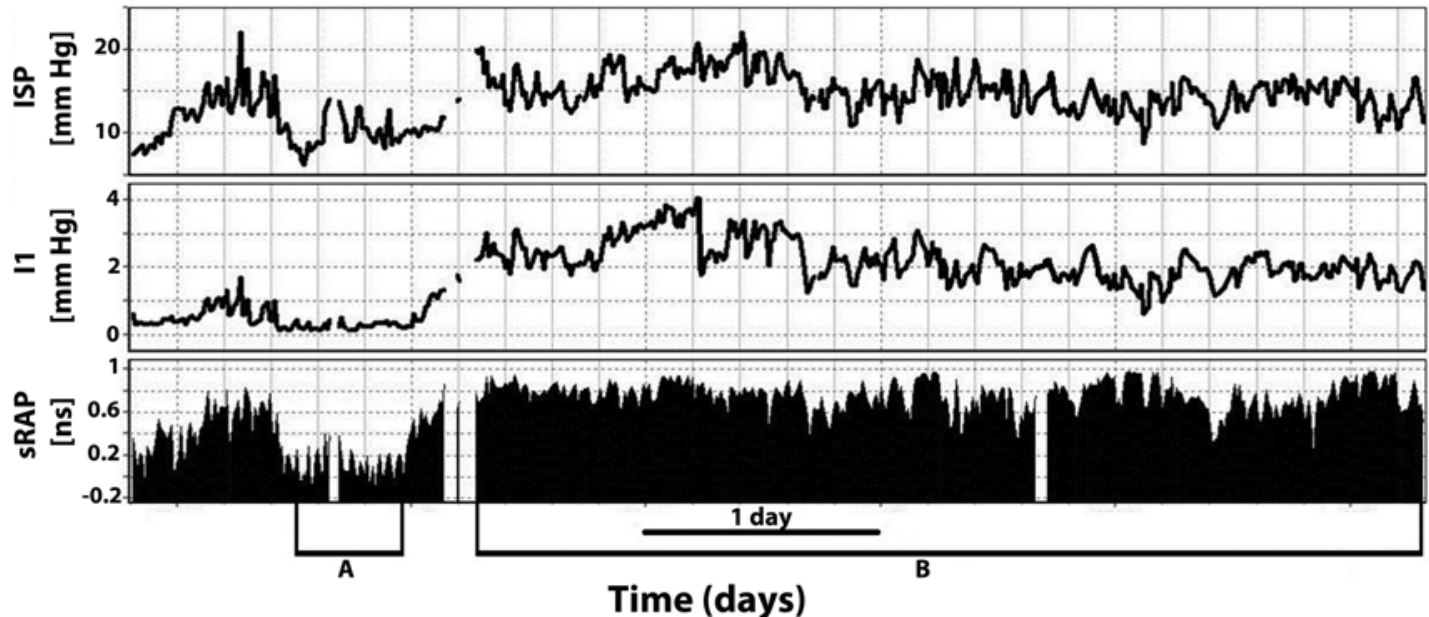

FIG. 4. Example of a long-term recording of ISP and its fundamental harmonic amplitude (I1) in a single patient. The relationship between ISP and I1 is depicted with the correlation coefficient index SRAP in order to represent the capacity of the spinal system for pressure-volume compensation. During period A, SRAP is at low levels, indicating a good compensatory reserve, whereas high SRAP during period $\mathbf{B}$ is indicative of a direct association between ISP and I1 and denotes an impaired compensatory capacity. 


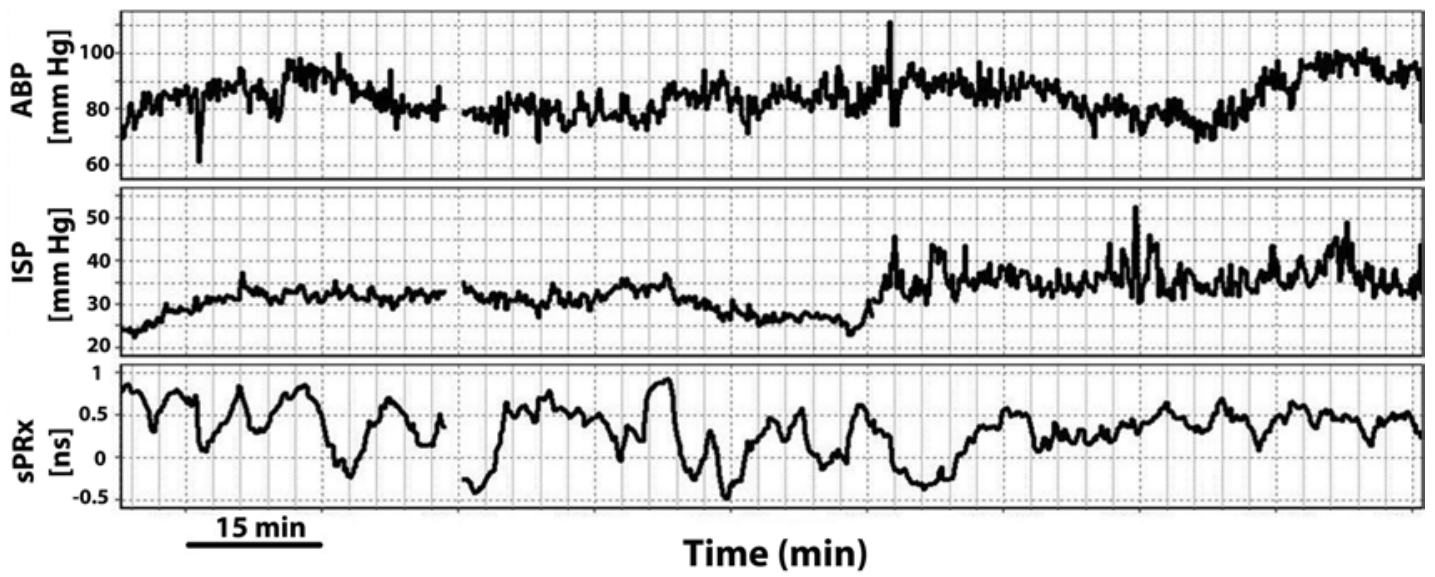

FIG. 5. Time series of ABP and ISP measurements in a recording of a single patient. The relationship between ABP and ISP can be depicted using the spinal pressure reactivity index (sPRx), which can present a measure of spinal vascular reactivity: 0 or negative sPRx indicates normal reactivity, whereas positive $\operatorname{sPRx}(>0.3)$ can signify an impairment in the capacity of the vessels to react to changes in pressure.

form, which is formed by the superimposition of the pulse components (P1, P2, and P3 peaks) and is synchronized with arterial pressure. The proportions between $\mathrm{P} 1$ and $\mathrm{P} 2$ vary, but the general trends indicate that as mean ISP increases, P2 becomes dominant and increases above P1. In some cases, this increase in P2 first causes the pulse wave configuration to acquire a round shape, with a further increase in mean ISP resulting in a triangular shape that is characteristic of both high ICP and ISP. On ICP analysis, the behavior of $\mathrm{P} 2$ has been known to depend on cerebral compliance, with the rise in ICP resulting in a reduction in compliance and a rise in P2. ${ }^{5}$ After TSCI, swelling of the injured cord results in obliteration of the subarachnoid space for CSF at that level, which is associated with a significant increase in ISP at the injury site ${ }^{25}$ because the lack of distensibility of the dura makes it unable to compensate for the expansion of the spinal cord tissue, like in an injured brain with brain edema. Therefore, further increases in ISP reduce spinal compliance, as reflected in a rise in $\mathrm{P} 2$.

Spectral analysis of the ISP signal revealed a frequency composition of ISP that is analogous to ICP, with the presence of slow, respiratory, and pulse waves. The pulse waves consist of multiple harmonics, with the fundamental harmonic amplitude of ISP at the heart rate frequency being proportional to the changes in the mean ISP value. This proportionality may suggest that the spinal pressurevolume relationship is exponential ${ }^{2,17}$-similar to the cranial compartment-and a characterization of both the elastance of the cerebrospinal space and the transmission of arterial pulsations to the CSF compartment. ${ }^{4}$

The sRAP index, as an equivalent of RAP, denotes the degree of correlation between mean ISP and its amplitude in short periods of time and can indicate the working point in the exponential pressure-volume curve. An sRAP close to 0 indicates a lack of synchronization between changes in the mean ISP and its amplitude, signifying a good compensatory reserve at low ISP, whereas a change in volume produces no or very little change in pressure. In contrast, as sRAP increases toward a value of +1 , a direct relationship between mean ISP and its amplitude is established. This is indicative of the passive association of changes between mean ISP and its amplitude and an impaired compensatory reserve, where a further change in volume cannot be compensated and will cause an increase in ISP.

In TBI, the association between the slow fluctuations of ABP and ICP, as expressed by the PRx index, can characterize the autoregulatory status of the cerebrovascular bed. Similarly, in SCI, the equivalent sPRx index can be cal-
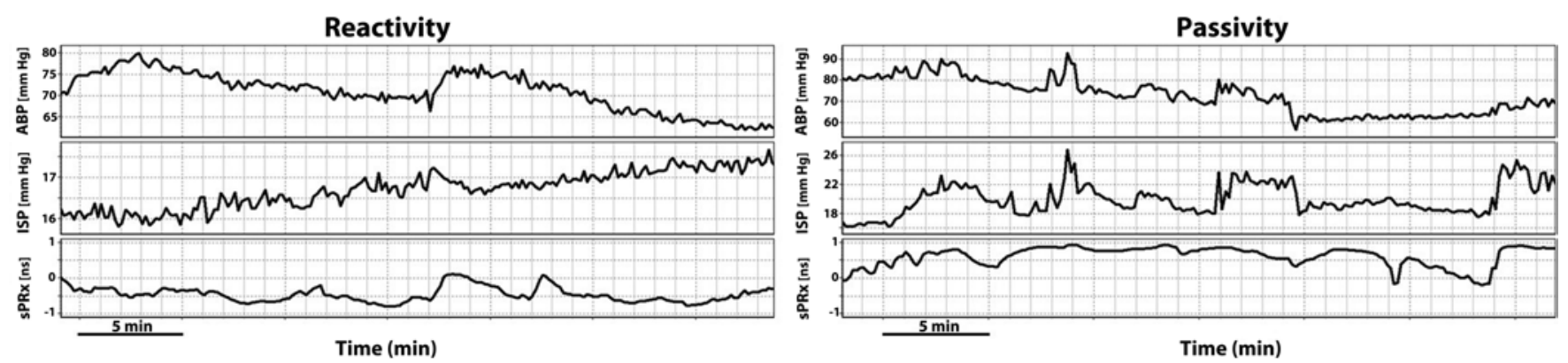

FIG. 6. Examples of assessing sPRx through the relationship between slow waves, ABP, and ISP in 2 different patients. Left: Most of the time sPRx is below 0 , indicating an inverse relationship between ABP and ISP and signifying the reactivity of the vascular bed to changes in pressure. Right: SPRx is highly positive, demonstrating a passive and direct relationship between ISP and $A B P$ and signifying impaired spinal vascular reactivity. 

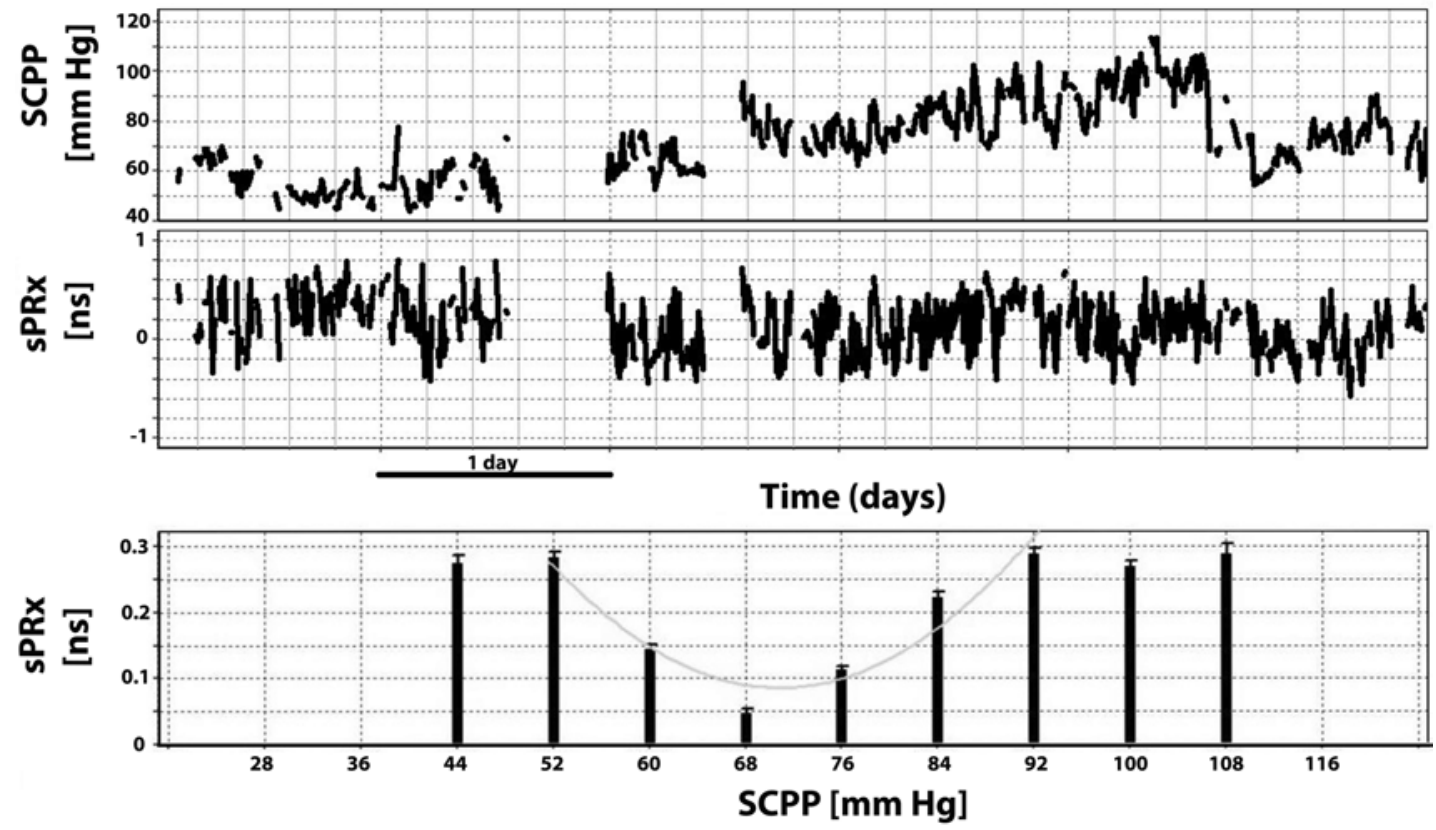

FIG. 7. Relationship between SCPP and SPRx in a patient after spinal cord injury. When plotted together, the relationship between SPRx and SCPP demonstrates a U-shaped curve, with autoregulatory capacity becoming worse at both low and high SCPP and optimal SCPP indicated by the lowest SPRx value.

culated using $\mathrm{ABP}$ and ISP measurements to specify the spinal vascular reactivity, which could be associated with the equivalent autoregulatory capacity for supplying blood to the spinal cord. Our recordings present long-time fluctuations in sPRx (Fig. 5) with periods of active spinal pressure reactivity where changes in $\mathrm{ABP}$ were compensated and rendered a negative sPRx (Fig. 6 left), or impaired reactivity where changes in ABP were passively transmitted to ISP and thereby rendered a positive sPRx (Fig. 6 right).

The relationship between SPRx and SCPP can reveal important aspects regarding how autoregulation is associated with the blood supply to the spinal cord. Both hypoand hyperperfusion could be linked with impaired spinal autoregulatory capacity, as shown by the U-shaped curve of sPRx plotted against SCPP (Fig. 7) which indicates that nonreactive vessels are unable to provide adequate perfusion to the spinal cord. Through this relationship, the optimum SCPP value for the management of TSCI patients could be characterized by the minimum sPRx. Likewise, according to TBI analysis and PRx, the philosophy behind calculating the "optimal SCPP" is that the minimum sPRx would indicate the specific point at which pressure reactivity is most effective. In that way, optimal SCPP could also be applied to TSCI patients, with adjustment of SCPP to its indicated optimal value providing adequate levels of perfusion to the spinal cord. Optimal SCPP should be individually calculated for each specific patient, as patterns of injury are highly variable and thus one SCPP threshold cannot be optimally applied to all patients, as is the case for TBI patients. ${ }^{1,24}$

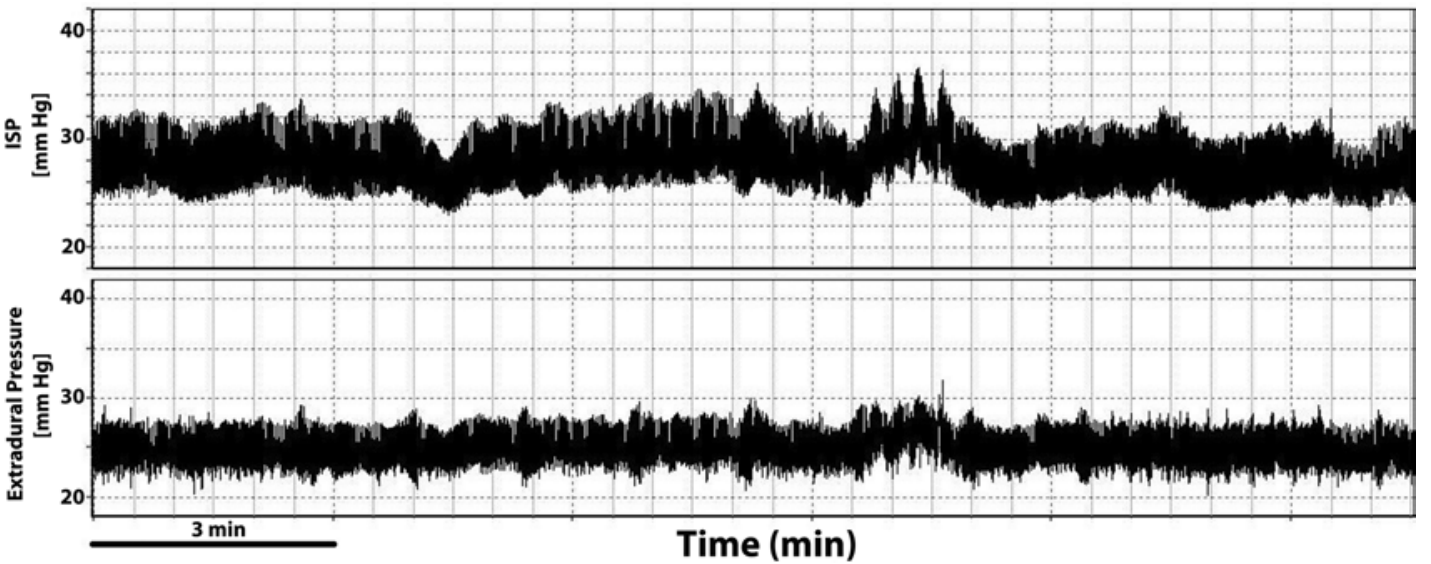

FIG. 8. Simultaneous monitoring of both subdural ISP and extradural pressure. Extradural pressure demonstrates similar behavior to ISP; however, extradural pressure appears to demonstrate overall lower values and slow waves of a smaller magnitude in comparison with ISP, possibly demonstrating a buffering role by the dura mater. 
In head injuries, a large difference between actual CPP and the optimal CPP value is thought to play an important role in terms of outcome: patients with significantly lower or higher CPP than the indicated optimal level demonstrate less favorable outcomes after TBI. ${ }^{1,24}$ A future study focusing on optimal SCPP and outcomes could confirm if this also applies to TSCI patients.

\section{Limitations}

The small number of patients who received ISP monitoring is a limitation of this study. Even though strong similarities are present between morphology and waveform analysis of ICP and ISP, a larger amount of data are required to potentially establish a subsequent link of ISP, SCPP, and their derived indices SRAP and SPRx with functional outcome following spinal cord injury. However, the purpose of this study was observational and to provide a qualitative illustration of ISP characteristics using more detailed continuation of our previous study. ${ }^{25}$

In terms of ICP monitoring, supratentorial pressure gradients may exist. Similar gradients may exist in ISP. Therefore, it is likely that the pressure measured within nonswollen sections of the spinal cord is much lower or even nearly normal. ${ }^{25}$ This is something that should be examined in future studies.

\section{Conclusions}

This preliminary study suggested that the methodology for analyzing the ICP waveform may be transformed to analyzing the ISP waveform, and the observational results indicated the morphological and spectral similarities between ISP in spinal cord injury and ICP recorded in TBI. More studies on this topic are needed to verify the clinical feasibility of applying the known methods of ICP waveform analysis, such as the indices of compensatory reserve and autoregulation, in patients with TSCI.

\section{Acknowledgments}

We thank T. Jones, M. Crocker, T. Bishop, J. Barnard (St. George's Hospital), and D. Bell (King's College Hospital).

\section{References}

1. Aries MJH, Czosnyka M, Budohoski KP, Steiner LA, Lavinio A, Kolias AG, et al: Continuous determination of optimal cerebral perfusion pressure in traumatic brain injury. Crit Care Med 40:2456-2463, 2012

2. Avezaat CJ, van Eijndhoven JH, Wyper DJ: Cerebrospinal fluid pulse pressure and intracranial volume-pressure relationships. J Neurol Neurosurg Psychiatry 42:687-700, 1979

3. Balestreri M, Czosnyka M, Steiner LA, Schmidt E, Smielewski P, Matta B, et al: Intracranial hypertension: what additional information can be derived from ICP waveform after head injury? Acta Neurochir (Wien) 146:131-141, 2004

4. Børgesen SE, Gjerris F: The predictive value of conductance to outflow of CSF in normal pressure hydrocephalus. Brain 105:65-86, 1982

5. Cardoso ER, Rowan JO, Galbraith S: Analysis of the cerebrospinal fluid pulse wave in intracranial pressure. J Neurosurg 59:817-821, 1983

6. Carrera E, Kim DJ, Castellani G, Zweifel C, Czosnyka Z,
Kasparowicz M, et al: What shapes pulse amplitude of intracranial pressure? J Neurotrauma 27:317-324, 2010

7. Czosnyka M, Guazzo E, Whitehouse M, Smielewski P, Czosnyka Z, Kirkpatrick P, et al: Significance of intracranial pressure waveform analysis after head injury. Acta Neurochir (Wien) 138:531-542, 1996

8. Czosnyka M, Hutchinson PJ, Balestreri M, Hiler M, Smielewski P, Pickard JD: Monitoring and interpretation of intracranial pressure after head injury, in Hoff JT, Keep RF, Xi G, et al (eds): Brain Edema XIII. Vienna: Springer, 2006, pp 114-118

9. Czosnyka M, Pickard JD: Monitoring and interpretation of intracranial pressure. J Neurol Neurosurg Psychiatry 75:813-821, 2004

10. Czosnyka M, Price DJ, Williamson M: Monitoring of cerebrospinal dynamics using continuous analysis of intracranial pressure and cerebral perfusion pressure in head injury. Acta Neurochir (Wien) 126:113-119, 1994

11. Czosnyka M, Richards HK, Czosnyka Z, Piechnik S, Pickard JD, Chir M: Vascular components of cerebrospinal fluid compensation. J Neurosurg 90:752-759, 1999

12. Czosnyka M, Smielewski P, Timofeev I, Lavinio A, Guazzo E, Hutchinson P, et al: Intracranial pressure: more than a number. Neurosurg Focus 22(5):E10, 2007

13. Davson H, Hollingsworth G, Segal MB: The mechanism of drainage of the cerebrospinal fluid. Brain 93:665-678, 1970

14. Gobiet W, Grote W, Bock WJ: The relation between intracranial pressure, mean arterial pressure and cerebral blood flow in patients with severe head injury. Acta Neurochir (Wien) 32:13-24, 1975

15. Howells T, Lewén A, Sköld MK, Ronne-Engström E, Enblad P: An evaluation of three measures of intracranial compliance in traumatic brain injury patients. Intensive Care Med 38:1061-1068, 2012

16. Kolias AG, Guilfoyle MR, Helmy A, Allanson J, Hutchinson PJ: Traumatic brain injury in adults. Pract Neurol 13:228235,2013

17. Löfgren J, von Essen C, Zwetnow NN: The pressure-volume curve of the cerebrospinal fluid space in dogs. Acta Neurol Scand 49:557-574, 1973

18. Lundberg N: Continuous recording and control of ventricular fluid pressure in neurosurgical practice. Acta Psychiatr Scand Suppl 36:1-193, 1960

19. Marmarou A, Maset AL, Ward JD, Choi S, Brooks D, Lutz $\mathrm{HA}$, et al: Contribution of CSF and vascular factors to elevation of ICP in severely head-injured patients. J Neurosurg 66: $883-890,1987$

20. Marmarou A, Shulman K, LaMorgese J: Compartmental analysis of compliance and outflow resistance of the cerebrospinal fluid system. J Neurosurg 43:523-534, 1975

21. Miller JD, Stanek A, Langfitt TW: Concepts of cerebral perfusion pressure and vascular compression during intracranial hypertension. Prog Brain Res 35:411-432, 1972

22. Pearce JMS: Queckenstedt's manoeuvre. J Neurol Neurosurg Psychiatry 77:728, 2006

23. Ryder HW, Espey FF, Kimbell FD, Penka EJ, Rosenauer A, Podolsky B, et al: The mechanism of the change in cerebrospinal fluid pressure following an induced change in the volume of the fluid space. J Lab Clin Med 41:428-435, 1953

24. Steiner LA, Czosnyka M, Piechnik SK, Smielewski P, Chatfield D, Menon DK, et al: Continuous monitoring of cerebrovascular pressure reactivity allows determination of optimal cerebral perfusion pressure in patients with traumatic brain injury. Crit Care Med 30:733-738, 2002

25. Werndle MC, Saadoun S, Phang I, Czosnyka M, Varsos GV, Czosnyka ZH, et al: Monitoring of spinal cord perfusion pressure in acute spinal cord injury: initial findings of the injured spinal cord pressure evaluation study. Crit Care Med 42:646-655, 2014 


\section{Disclosure}

Mr. Varsos is supported by an A.G. Leventis Foundation Scholarship and a Charter Studentship from St. Edmund's College, Cambridge, United Kingdom. Dr. Kolias is supported by a Royal College of Surgeons of England Research Fellowship, a National Institute for Health Research (NIHR) Academic Clinical Fellowship, and a Raymond and Beverly Sackler Studentship. Dr. Werndle is supported by the UK Spinal Cord Injuries Research Network (UKSCIRN), a Royal College of Surgeons of England Research Fellowship, and the Housham Fellowship from the Neurosciences Research Foundation. Dr. Papadopoulos is supported by the UKSCIRN and the Guthy Jackson Charitable Foundation, and is also funded by the Wings for Life Spinal Cord Research Foundation. Dr. Czosnyka is supported by NIHR Cambridge Biomedical Center. Drs. Czosnyka and Smielewski have a financial interest in a fraction of the licensing fee for ICM+Software, which is licensed by Cambridge Enterprise, Cambridge, United Kingdom, and used in this study.

\section{Author Contributions}

Conception and design: Papadopoulos. Acquisition of data: Werndle, Phang, Saadoun, Zoumprouli, Papadopoulos. Analysis and interpretation of data: Varsos. Drafting the article: Varsos. Critically revising the article: Werndle, Z Czosnyka, Smielewski, Kolias, Phang, Saadoun, Bell, Zoumprouli, Papadopoulos, M Czosnyka. Reviewed submitted version of manuscript: Z Czosnyka, Smielewski, M Czosnyka. Approved the final version of the manuscript on behalf of all authors: Varsos.

Administrative/technical/material support: Werndle, Smielewski, Phang, Saadoun, Zoumprouli. Study supervision: Papadopoulos, M Czosnyka.

\section{Correspondence}

Georgios V. Varsos, St Edmund's College, University of Cambridge, Cambridge, CB3 0BN, United Kingdom. email: gv249@ cam.ac.uk. 